\title{
Factores Psicosociales Que Intervienen En El Rendimiento Académico Estudio De Caso: Análisis A Estudiantes De La Carrera De Cultura Física Y Entrenamiento Deportivo
}

\author{
César Mario Rodríguez Sotomayor
}

Magister en docencia, mención intervención psicopedagógica

Mg. Darío Javier Díaz Muñoz

Mg. Víctor Daniel Aldas Rovayo

Docentes de la Universidad Técnica de Ambato - Ecuador

Mg. Orlando David Mazón Moreno

Docente de la Escuela Superior Politécnica de Chimborazo, Ecuador

\section{Hernán Leopoldo Ponce Bravo PhD}

Actividad física y salud

Docente de la Universidad Nacional de Chimborazo, Ecuador

Doi: 10.19044/esj.2017.v13n29p317 URL:http://dx.doi.org/10.19044/esj.2017.v13n29p317

\begin{abstract}
The objective of the present research was to analyze the psychosocial factors that intervene in the academic performance of the students in the race of physical culture and sports training of the "Universidad Nacional de Chimborazo" in the academic period from September 2014 to February 2015. The study sample was 72 students of a population of 97 students, 57 male and 15 female. The methodology of this study uses a cross-sectional research, quasi-experimental, qualitative-quantitative, bibliographic, and field research. Based on the collection of data, the instruments used were the academic record. A questionnaire was developed which served to identify the psychosocial factors of the students. Among the most relevant results, it was evidenced that out of 97 students corresponding to $100 \%$, only 25 , that is $25.77 \%$, exceeded the required score to approve the semester. Therefore, 72 students representing $74.22 \%$ obtained lower score than required for approval. In conclusion, it was obtained that the psychosocial factors that influenced the academic performance of the students were: the always consumption of cigarettes representing $25 \%$. On the other hand, $55.56 \%$ had difficulty in sometimes distributing their time adequately for educational tasks.
\end{abstract}


Keywords: Psychosocial factors, academic performance, analysis, psycho pedagogical proposal

\section{Resumen}

El objetivo de la presente investigación fue analizar los factores psicosociales que intervienen en el rendimiento académico de los estudiantes de la carrera de cultura física y entrenamiento deportivo de la Universidad Nacional de Chimborazo, en el período académico septiembre 2014 febrero 2015, la muestra en estudio fue de 72 estudiantes, de una población de 97 estudiantes, 57 de género masculino y 15 de género femenino. Para la metodología se aplicó una investigación transversal, cuasi-experimental, cuali-cuantitativa, bibliográfica y de campo; para la recolección de datos los instrumentos empleados fueron el record académico y se elaboró un cuestionario que sirvió para identificar los factores psicosociales de los estudiantes. Entre los resultados más relevantes se evidenció que de 97 estudiantes correspondientes al 100\%, solo 25, es decir el 25,77\% superaron el puntaje requerido para aprobar el semestre, por tanto 72 estudiantes que representan el $74,22 \%$ obtuvieron un puntaje inferior al requerido para su aprobación. Como conclusión se obtuvo que los factores psicosociales que influyeron en el rendimiento académico de los estudiantes fueron: el consumo siempre de cigarrillo representando el 25\%, mientras que el 55,56\% tuvo dificultad a veces de distribuir adecuadamente su tiempo para las tareas educativas.

Palabras-claves: Factores psicosociales, rendimiento académico, análisis, propuesta psicopedagógica

\section{Introducción}

En América Latina en los últimos 25 años se han realizado alrededor de una centena de investigaciones que tratan de identificar las variables del rendimiento. Existen una amplia literatura sobre los factores que afectan el mismo, reconocen ampliamente que uno de los determinantes esenciales es la familia y sus características sociodemográficas, sin dejar de lado la controversia sobre el efecto especifico de otros factores como el nivel de gasto, relaciones de los profesores y compañeros, lo que se denomina como insumos del proceso educativo, los mismos que contribuyen a la adquisición de habilidades cognitivas, independientemente de las características del núcleo familiar. Otras relaciones positivas, incluyen; nivel de satisfacción con el método de evaluación, genero de los alumnos, horas de estudio a la semana y la edad de los mismos (Abarca \& Sánchez, 2005). 
Sumado a este estudio Cascón (2000), expuso los factores que influyen en el rendimiento académico de los jóvenes de latinoamérica, los resultados que obtuvo sugieren que la pertenencia institucional y el involucramiento de la familia en especial los padres en los procesos de educación, son elementos de protección que promueven el rendimiento académico de adolescentes latinos. En cuanto al apoyo familiar al autor señala que existe evidencia de que los padres que ofrecen apoyo a sus hijos logran que estos tengan un mejor rendimiento académico al incrementar sus niveles de seguridad emocional y satisfacción personal.

Los autores Fernández, Martínez, y Melipillán (2009), entienden por rendimiento académico a una medida, determinada por resultados de pruebas, exámenes o calificaciones, que representan un desempeño exitoso ya sea en la infancia o adolescencia, sostiene que es una respuesta que encaja dentro de 2 categorías habilidad y esfuerzo, habilidad talento y esfuerzo energía dependiendo de las variables sociodemográficas, así como también el resultado de experiencias pasadas.

En otros términos, el rendimiento académico es una medida de las capacidades del alumno, que dice lo que éste ha asimilado en el transcurso del proceso instructivo y formativo. Asimismo presume la capacidad del alumno para enfrentar a los estímulos educativos. En este aspecto, el rendimiento académico está vinculado a nuestras aptitudes.

En el sistema educativo ecuatoriano, en especial en las universidades, la mayor parte de las calificaciones se basan en el sistema decimal, es decir de 0 a 10. Sistema en el cual el puntaje obtenido se traduce a la categorización del logro del aprendizaje. Un estudio realizado sobre las carreras que el país más necesita, desde el punto de vista de los estudiantes que cursan el pre-universitario, se obtuvieron los siguientes resultados: Medicina (26.8\%), Economía (20.4\%), Ingeniería Comercial y Empresarial (17,2\%), e Ingeniería de Sistemas y Computación (7,52\%). Consejo Nacional de Educación Superior (CONESUP, 2015).

En el Ecuador los estudiantes tienen la oportunidad de ingresar al SNNA (Sistema Nacional de Nivelación y Admisión), cuyo objetivo garantiza la igualdad de oportunidades, transparencia y acceso a la educación superior. El sistema que sin duda es perfectible, busca solucionar los problemas que cada año se presentaban, con esto se pretende disminuir o eliminar la deserción estudiantil.

La investigación se desarrolló en la "Universidad Nacional de Chimborazo", ubicada en la ciudad de Riobamba - Ecuador. La población en estudio fue de 97 estudiantes, de la carrera de Cultura Física y Entrenamiento Deportivo, de la Facultad de Ciencias de la Salud legalmente matriculados que asistieron normalmente durante el período académico Septiembre 2014 Febrero 2015. 


\section{Objetivos de la Investigación}

- Identificar los factores psicológicos prevalentes que influyen en el rendimiento académico de los estudiantes de la carrera de Cultura Física y Entrenamiento Deportivo.

- Determinar los factores sociales relevantes que repercuten en el Rendimiento Académico.

- $\quad$ Conocer los promedios de rendimiento académico de los estudiantes de la carrera de Cultura Física, para establecer rangos de rendimiento.

\section{Metodología}

\section{Diseño de la Investigación}

Para responder sobre el diseño de la investigación se tomó en cuenta lo siguiente:

Transversal.- Estudio que se realizó en un momento determinado de tiempo que fue el período académico Septiembre de 2014 a Febrero 2015.

Cuasi - experimental.- Por medio de este tipo de investigación que permitió aproximarse a los resultados de una indagación experimental en escenarios en las que no es factible el control y manejo dominantes de las variables.

\section{Técnicas e Instrumentos Para Recolección de Datos Técnicas}

Encuesta.- Mediante esta técnica se pudo conocer los pensamientos, actitudes y los comportamientos de los encuestados, sin modificar el contexto ni el fenómeno donde se recoge la información ya sea para entregarlo en forma estadísticas a través de cuadros y gráficos. Los datos se obtienen realizando un conjunto de preguntas normalizadas.

Observación.- Consistió en observar, acumular e interpretar los acontecimientos y comportamientos, tal como se realizan habitualmente, se buscó contemplar de forma cuidadosa y sistemática de todas aquellas características presentadas en un contexto sin que éstas sean manipuladas (Sierra, 1994).

\section{Instrumentos}

Cuestionario.- $\mathrm{Al}$ ser un instrumento de investigación que consiste en una serie de preguntas y otras indicaciones, cumplió con el propósito de obtener información de los consultados, se planteó de forma organizada y práctica en busca de una opinión generalizada de un tema específico.

El cuestionario fuer elaborado por el grupo de investigación para identificar los factores psicosociales que influyeron en el rendimiento académico de los estudiantes investigados. Consta de 15 preguntas diferenciadas por: 
- Datos Generales

- Factores Psicológicos

- Factores Sociales

- Factores Académicos

Récord académico.- Este instrumento registra las calificaciones de todas las asignaturas del estudiante, de acuerdo a la malla curricular que posee cada carrera. Tiene un formato establecido por la Unidad de Planificación Académica de la Universidad.

\section{Población}

La carrera de Cultura Física y Entrenamiento Deportivo, de la Facultad de Ciencias de la Salud, cuenta en el período académico Septiembre 2014 - Febrero 2015, con 97 estudiantes legamente matriculados de los cuales 72 tuvieron que rendir evaluaciones supletorias.

\section{Muestra}

Para la selección de la respectiva muestra se tomó en cuenta a los 72 estudiantes, que tuvieron que rendir las evaluaciones supletorias.

\section{Procedimiento Para el Análisis e Interpretación de Resultados}

En el procedimiento para el procesamiento de datos, fueron analizados en forma cualitativa y cuantitativamente a través de los instrumentos analizados, luego de la respectiva tabulación, se estableció su representación mediante cuadros y gráficos (pasteles y barras) expresados en porcentajes correspondientes en el sistema Excel.

\section{Resultados de la Investigación}

Análisis e interpretación de resultados obtenidos mediante la aplicación de la encuesta a los estudiantes de la carrera de cultura física, antes del programa de intervención psicopedagógica

\section{Datos Generales}

Tabla 1. Género del estudiante

\begin{tabular}{|c|c|c|}
\hline Género & $\mathrm{Fa}$ & Fr \\
\hline MASCULINO & 57 & $79,17 \%$ \\
\hline FEMENINO & 15 & $20,83 \%$ \\
\hline TOTAL & 72 & $100 \%$ \\
\hline
\end{tabular}

Fuente: Encuesta aplicada a los estudiantes de la Carrera de Cultura Física y Entrenamiento Deportivo de la UNACH Realizado por: equipo de investigación 


\section{Interpretación}

Esto nos conlleva a determinar que en la carrera de Cultura Física y Entrenamiento Deportivo, existe más la presencia de estudiantes varones (57) que de mujeres (15).

Factores Psicológicos

Tabla 2. ¿Cuántas horas duerme?

\begin{tabular}{|c|c|c|}
\hline Horas de sueño & Fa & Fr \\
\hline Más de 8 horas & 9 & $12,50 \%$ \\
\hline Entre 6 a 8 horas & 46 & $63,88 \%$ \\
\hline Menos de 6 horas & 17 & $23,61 \%$ \\
\hline TOTAL & 72 & $100 \%$ \\
\hline
\end{tabular}

Fuente: Encuesta aplicada a los estudiantes de la Carrera de Cultura Física y Entrenamiento Deportivo de la UNACH

Realizado por: equipo de investigación

\section{Interpretación}

Según la organización mundial de la salud (OMS) insiste en que dormir no es un placer sino una necesidad. Por lo que recomienda dormir por lo menos 6 horas diarias, puesto que si no se cumple este tiempo, se produce la reducción de los reflejos, capacidad de concentración reducida y dificultades de deseo y digestivos, son aquellas secuelas de un insuficiente descanso.

Tabla 3. ¿Usted ingiere alcohol para aliviar la tensión o los nervios?

\begin{tabular}{|c|c|c|}
\hline Intensidad de Consumo & Fa & Fr \\
\hline Siempre & 15 & $20,83 \%$ \\
\hline A veces & 20 & $27,78 \%$ \\
\hline Nunca & 37 & $51,39 \%$ \\
\hline Total & 72 & $100 \%$ \\
\hline
\end{tabular}

Fuente: Encuesta aplicada a los estudiantes de la Carrera de Cultura Física y Entrenamiento Deportivo de la UNACH

Realizado por: equipo de investigación

\section{Interpretación}

Este resultado demuestra que el porcentaje de consumo de alcohol es representativamente alto ya que si se suman aquellos que dicen haber consumido una vez a más veces si tiene que casi la mitad del universo está influenciado por esta conducta poco recomendable en las personas. 
Tabla 4. ¿Con que frecuencia usted consume cigarrillos?

\begin{tabular}{|c|c|c|}
\hline Intensidad de Consumo & $\mathrm{Fa}$ & $\mathrm{Fr}$ \\
\hline Siempre & 18 & $25,00 \%$ \\
\hline A veces & 32 & $44,44 \%$ \\
\hline Nunca & 22 & $30,56 \%$ \\
\hline Total & 72 & $100 \%$ \\
\hline
\end{tabular}

Fuente: Encuesta aplicada a los estudiantes de la Carrera de Cultura Física y Entrenamiento Deportivo de la UNACH

Realizado por: equipo de investigación

\section{Interpretación}

El problema del tabaquismo, se ha convertido en un gran inconveniente en la población y en diferentes grupos de edad, pero principalmente en la juventud, los cuales por razones indebidamente justificadas, se han visto involucrados en este lamentable vicio, el cual desencadena en problemas de salud, como el cáncer de pulmón, mortalidad prematura, cataratas, neumonía, ataques cardíacos, asma, entre otros; aspectos que tienen efectos negativos y llega a presentar alteraciones en los estilos de vida del ser humano.

Tabla 5. ¿Qué sentimientos, emociones o pensamientos predominan en usted?

\begin{tabular}{|c|c|c|}
\hline Sentimientos/emociones/pensamientos & $\mathrm{Fa}$ & $\mathrm{Fr}$ \\
\hline Soledad y tristeza & 12 & $16,67 \%$ \\
\hline Irritabilidad & 35 & $48,61 \%$ \\
\hline Actitudes y pensamientos negativos & 10 & $13,88 \%$ \\
\hline Inseguridad Personal & 15 & $20,84 \%$ \\
\hline TOTAL & 72 & $100 \%$ \\
\hline
\end{tabular}

Fuente: Encuesta aplicada a los estudiantes de la Carrera de Cultura Física y Entrenamiento Deportivo de la UNACH

Realizado por: equipo de investigación

\section{Interpretación}

Debemos comprender que todos estos aspectos pueden conllevar a la depresión, la cual es considerada como una enfermedad o trastorno mental que se caracteriza por una profunda tristeza, quebranto moral, autoestima baja, merma de interés por todo y baja de las funciones mentales. Estos aspectos pueden incidir notablemente en el rendimiento académico de los estudiantes. 
Tabla 6. ¿Qué molestias tiene durante la jornada académica?

\begin{tabular}{|c|c|c|}
\hline Molestias & $\mathrm{Si}$ & $\%$ \\
\hline Cabeza & 20 & $27,78 \%$ \\
\hline Estómago & 6 & $8,33 \%$ \\
\hline Espalda & 13 & $18,06 \%$ \\
\hline Hombros & 5 & $6,94 \%$ \\
\hline Cuello & 20 & $27,78 \%$ \\
\hline Dolores combinados & 8 & $11,11 \%$ \\
\hline TOTAL & 72 & $100 \%$ \\
\hline
\end{tabular}

Fuente: Encuesta aplicada a los estudiantes de la Carrera de Cultura Física y Entrenamiento Deportivo de la UNACH

Realizado por: equipo de investigación

\section{Interpretación}

La ansiedad es fundamentalmente un componente defensivo. Es una contestación de alarma ante circunstancias pensadas como amenazantes, entre ellas tenemos diferentes tipos de dolencias de carácter fisiológicas, como el dolor de cabeza, cuello, espalda, etc.

\section{Factores Sociales}

Tabla 7. ¿Usted con quien vive?

\begin{tabular}{|c|c|c|}
\hline Vive & $\mathrm{Fa}$ & Fr \\
\hline Familia & 45 & $62,50 \%$ \\
\hline Amigos & 9 & $12,50 \%$ \\
\hline Solo & 18 & $25,00 \%$ \\
\hline TOTAL & 72 & $100 \%$ \\
\hline
\end{tabular}

Fuente: Encuesta aplicada a los estudiantes de la Carrera de Cultura Física y Entrenamiento Deportivo de la UNACH

Realizado por: equipo de investigación

\section{Interpretación}

Al carecer de centros de educación superior en el lugar de residencia $\mathrm{u}$ otras opciones para mejorar los niveles de vida de las familias, desencadena en que poblaciones relativamente jóvenes tengan que movilizarse fuera de su ciudad habitual, conllevando a problemas sociales y el quebrantamiento familiar.

Tabla 8. ¿Para continuar sus estudios superiores tuvo que emigrar?

\begin{tabular}{|c|c|c|}
\hline Emigrar & $\mathrm{Fa}$ & $\mathrm{Fr}$ \\
\hline SI & 26 & $36,11 \%$ \\
\hline NO & 46 & $63,89 \%$ \\
\hline TOTAL & 72 & $100 \%$ \\
\hline
\end{tabular}

Fuente: Encuesta aplicada a los estudiantes de la Carrera de Cultura Física y Entrenamiento Deportivo de la UNACH

Realizado por: equipo de investigación 


\section{Interpretación}

Por el nuevo sistema de admisión de los estudiantes para optar por los diferentes centros de educación superior y también por la oferta académica propuesta en la actualidad, hace que exista un porcentaje considerable de estudiantes que por una necesidad de preparación, tienen que emigrar con el objetivo de alcanzar su profesionalización dentro de las diversas carreras.

Tabla 9. ¿Tiene conflictos familiares que afectan al desarrollo de su estudio?

\begin{tabular}{|c|c|c|}
\hline Conflictos Familiares & $\mathrm{Fa}$ & $\mathrm{Fr}$ \\
\hline Siempre & 8 & $11,11 \%$ \\
\hline A veces & 24 & $33,33 \%$ \\
\hline Nunca & 40 & $55,56 \%$ \\
\hline TOTAL & 72 & $100 \%$ \\
\hline
\end{tabular}

Fuente: Encuesta aplicada a los estudiantes de la Carrera de Cultura Física y Entrenamiento Deportivo de UNACH

Realizado por: equipo de investigación

\section{Interpretación}

En vista que el rendimiento académico de los estudiantes de la carrera de Cultura Física no resulta satisfactorio, resultaba necesario investigar sobre los principales factores psicosociales que se encuentran interviniendo en su proceso académico; entre las dificultades de aprendizaje tienen desajustes emocionales, sociales, personales, de conducta.

Tabla 10. ¿Con que frecuencia usted tiene problemas económicos?

\begin{tabular}{|c|c|c|}
\hline Problemas Económicos & $\mathrm{Fa}$ & Fr \\
\hline Siempre & 17 & $23,61 \%$ \\
\hline A veces & 47 & $65,28 \%$ \\
\hline Nunca & 8 & $11,11 \%$ \\
\hline TOTAL & 72 & $100 \%$ \\
\hline
\end{tabular}

Fuente: Encuesta aplicada a los estudiantes de la Carrera de Cultura Física y Entrenamiento

Deportivo de la UNACH

Realizado por: equipo de investigación

\section{Interpretación}

La realidad financiera del entorno social y familiar trasciende en el alumno cuando no se presentan las condiciones necesarias para desarrollar sin mayores dificultades la actividad educativa, puesto que requieren de aspectos esenciales como la residencia, transporte, alimentación, incluyendo gastos extras que se puedan presentar. Muchos de los estudiantes por la actual crisis económica, ayudan de alguna manera a los progenitores, de acuerdo a la disponibilidad de tiempo, principalmente en sectores del comercio, agricultura, entre otras. 
Factores Académicos

Tabla 11. ¿Usted considera que tiene excesivas tareas en el estudio?

\begin{tabular}{|c|c|c|}
\hline Tareas excesivas & $\mathrm{Fa}$ & $\mathrm{Fr}$ \\
\hline Siempre & 12 & $16,67 \%$ \\
\hline A veces & 54 & $75,00 \%$ \\
\hline Nunca & 6 & $8,33 \%$ \\
\hline TOTAL & 72 & $100 \%$ \\
\hline
\end{tabular}

Fuente: Encuesta aplicada a los estudiantes de la Carrera de Cultura Física y Entrenamiento

Deportivo de la UNACH

Realizado por: equipo de investigación

\section{Interpretación}

Dentro del proceso académico que se desarrolla en cada uno de los períodos, se desarrolla una serie de actividades entre las cuales mencionamos actividades de aprendizaje, entre los cuales están actividades de aprendizaje colaborativo, de prácticas de aplicación y experimentación y de trabajo autónomo, lo que implica un trabajo que demanda de más dedicación.

Tabla 12. ¿Considera usted que existen asignaturas con falta de motivación?

\begin{tabular}{|c|c|c|}
\hline Falta Motivación & $\mathrm{Fa}$ & $\mathrm{Fr}$ \\
\hline SI & 48 & $66,67 \%$ \\
\hline NO & 24 & $33,33 \%$ \\
\hline TOTAL & 72 & $100 \%$ \\
\hline
\end{tabular}

Fuente: Encuesta aplicada a los estudiantes de la Carrera de Cultura Física y Entrenamiento Deportivo de la UNACH

Realizado por: equipo de investigación

\section{Interpretación}

Entre los elementos que son determinantes para que un alumno no obtenga resultados satisfactorios, se hallan el problema de los contenidos programáticos, la falta de motivación para desarrollar sus estudios, la falta de comprensión de la información y la corrección subjetiva del docente, etc.

Tabla 13. ¿Usted distribuye adecuadamente su tiempo para las tareas educativas?

\begin{tabular}{|c|c|c|}
\hline Distribución adecuada del tiempo & $\mathrm{Fa}$ & $\mathrm{Fr}$ \\
\hline Siempre & 23 & $31,94 \%$ \\
\hline A veces & 40 & $55,56 \%$ \\
\hline Nunca & 9 & $12,50 \%$ \\
\hline TOTAL & 72 & $100 \%$ \\
\hline
\end{tabular}

Fuente: Encuesta aplicada a los estudiantes de la Carrera de Cultura Física y Entrenamiento

Deportivo de la UNACH

Realizado por: equipo de investigación 


\section{Interpretación}

El inconveniente que tiene el estudiante radica en la mala distribución del tiempo, por lo que no tiene un régimen o un hábito principalmente de estudio, lo cual no le permite desarrollar en forma adecuada todas las actividades académicas.

Tabla 14. ¿Tiene dificultades de memorización en el proceso de aprendizaje?

\begin{tabular}{|c|c|c|}
\hline Dificultades & $\mathrm{Fa}$ & $\mathrm{Fr}$ \\
\hline Siempre & 10 & $13,89 \%$ \\
\hline A veces & 44 & $61,11 \%$ \\
\hline Nunca & 18 & $25,00 \%$ \\
\hline TOTAL & 72 & $100 \%$ \\
\hline
\end{tabular}

Fuente: Encuesta aplicada a los estudiantes de la Carrera de Cultura Física y Entrenamiento Deportivo de la UNACH

Realizado por: equipo de investigación

\section{Interpretación}

La ansiedad en los estudiantes afecta significativamente su rendimiento académico, las personas que tienden a ser más ansiosas codifican menos la información, perdiendo la capacidad de memoria, debido angustias y preocupaciones internas que generalmente acarrean dentro de su proceso académico.

Tabla 15. ¿Le gustaría recibir apoyo psicológico?

\begin{tabular}{|c|c|c|}
\hline Apoyo Psicológico & $\mathrm{Fa}$ & $\mathrm{Fr}$ \\
\hline SI & 52 & $72,22 \%$ \\
\hline NO & 20 & $27,78 \%$ \\
\hline TOTAL & 72 & $100 \%$ \\
\hline
\end{tabular}

Fuente: Encuesta aplicada a los estudiantes de la Carrera de Cultura Física y Entrenamiento Deportivo de la UNACH

Realizado por: equipo de investigación

\section{Interpretación}

Para el desarrollo de las actividades académicas, se debe no tomar en cuenta solamente la parte cognitiva del estudiante, sino también complementarle con el aspecto psicológico, porque debemos entender que en la actualidad, la educación constituye un proceso integral y el aspecto psicológico desempeña un factor fundamental dentro del proceso académico. 
Análisis de resultados obtenidos antes y después del programa de intervención psicopedagógica Factores Psicológicos

Gráfico 1.

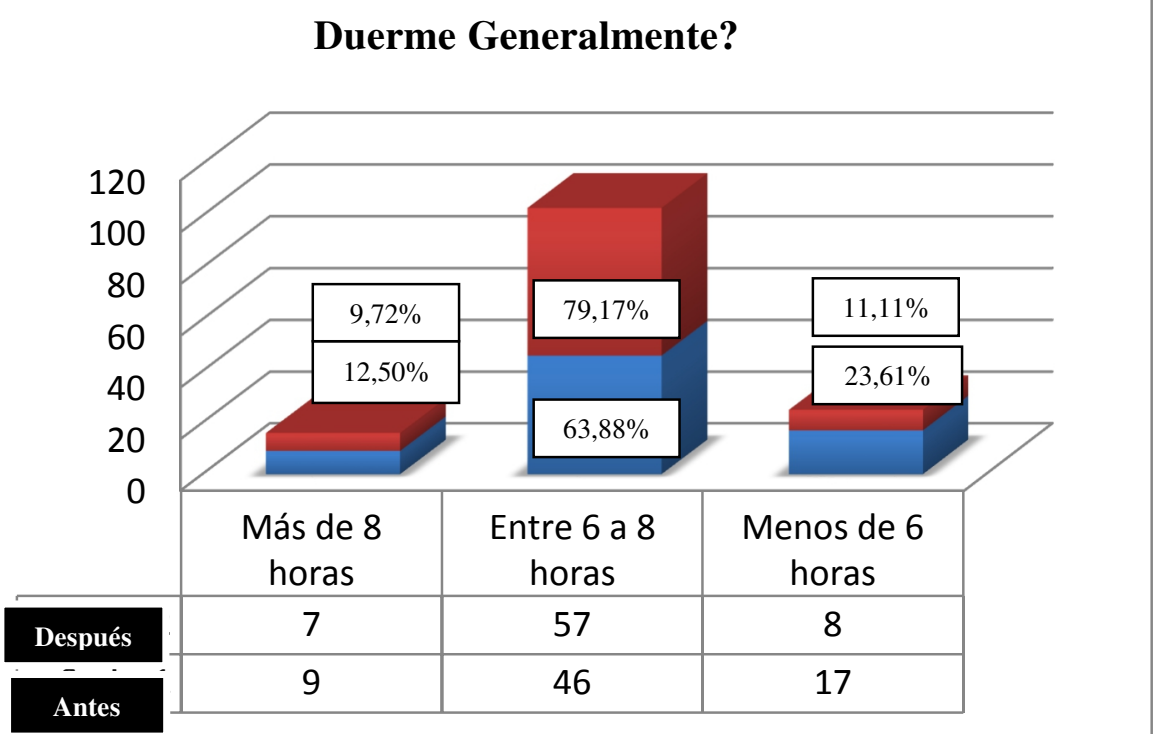

Fuente: Encuesta aplicada a los estudiantes de la Carrera de Cultura Física y Entrenamiento Deportivo de la UNACH

Realizado por: equipo de investigación

\section{Análisis}

En base al descanso de los estudiantes, antes de más de 8 horas, dormían en un $12,50 \%$, luego de la aplicación del Programa de Intervención duermen el 9,72\%; entre 6 y 8 horas, antes dormían un 63,88\%, después de la aplicación de talleres el 79,17\%; y menos de 6 horas, antes el 23,61\% y posterior a la aplicación el $11,11 \%$. 
Gráfico 2.

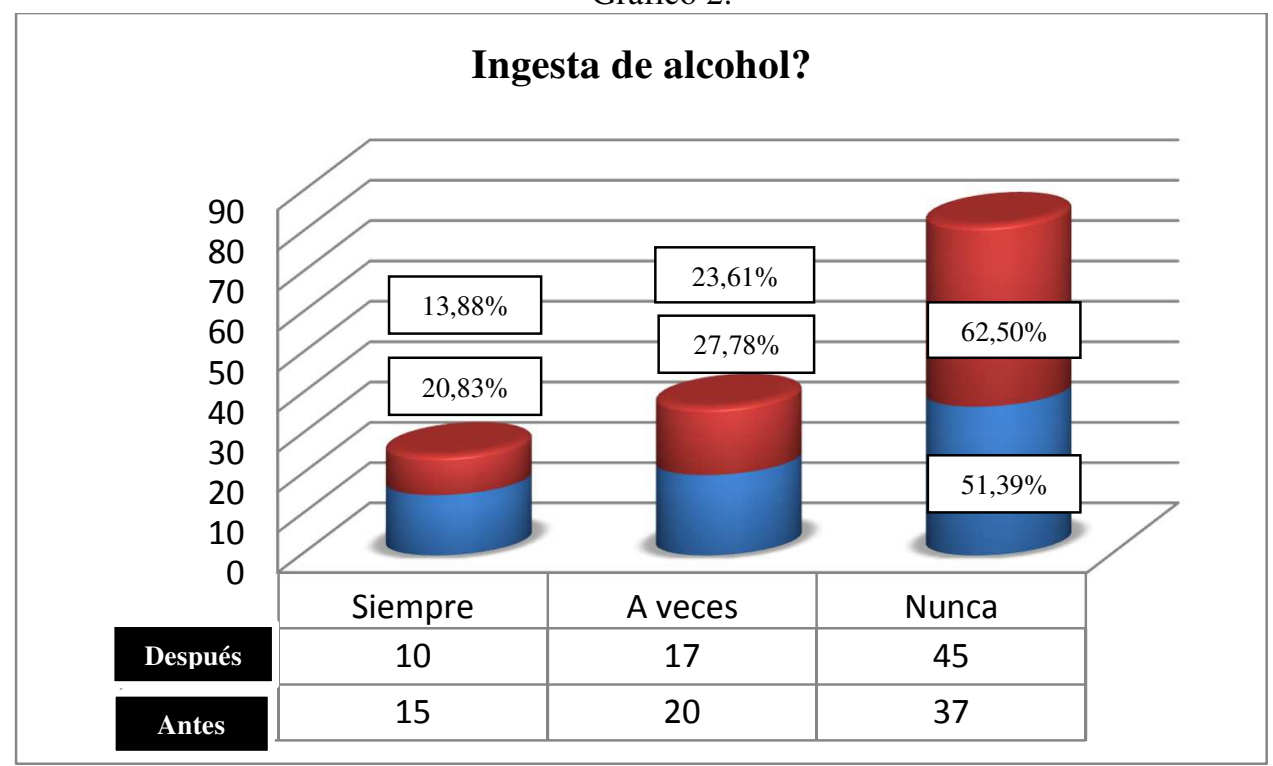

Fuente: Encuesta aplicada a los estudiantes de la Carrera de Cultura Física y Entrenamiento Deportivo de la UNACH

Realizado por: equipo de investigación

\section{Análisis}

De la población investigada sobre la ingesta de alcohol, ingerían Siempre el 20,83\% después de los talleres de Intervención el 13,88\%; A veces, antes el 27,78\%, después el 23,61\%; y Nunca, antes el $51,39 \%$ y posterior el $62,50 \%$. 
Gráfico 3.

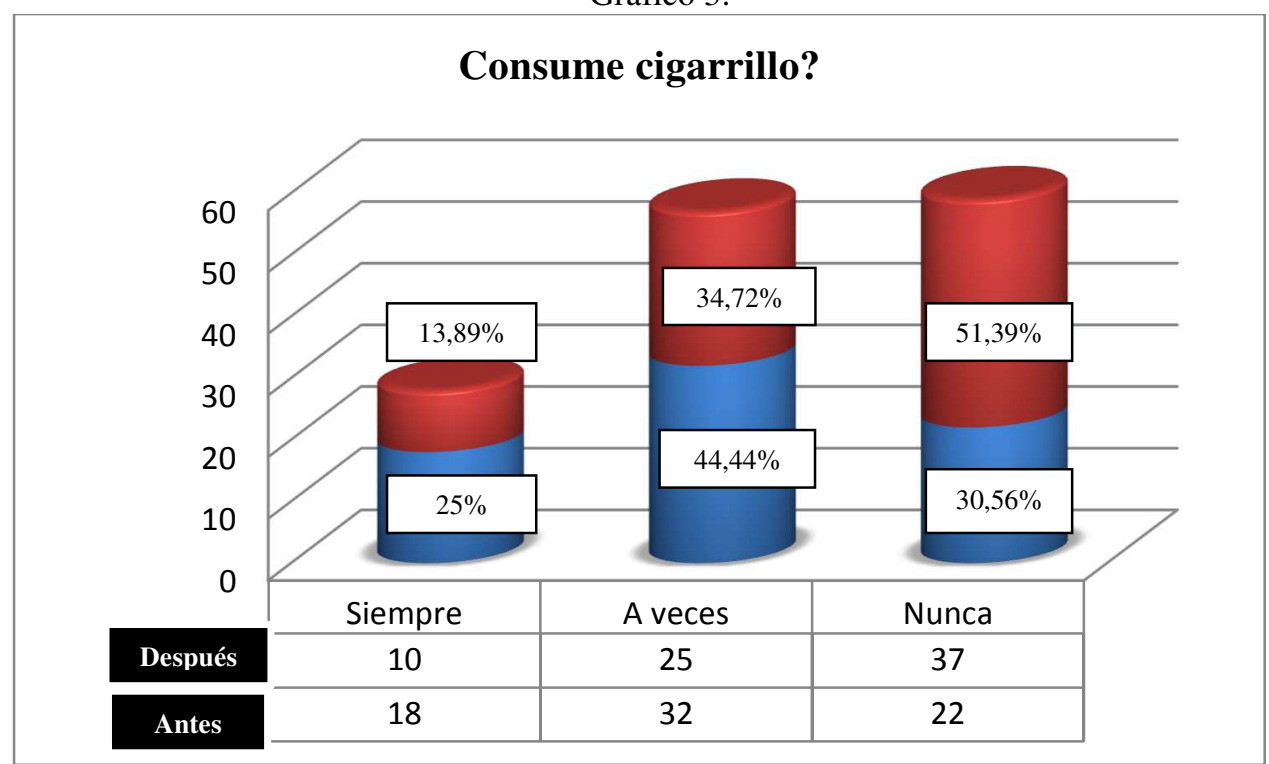

Fuente: Encuesta aplicada a los estudiantes de la Carrera de Cultura Física y Entrenamiento Deportivo de la UNACH

Realizado por: equipo de investigación

\section{Análisis}

En lo respecta a la utilización del cigarrillo, Siempre consumían el $25 \%$, luego de la Intervención el 13,89\%; A veces consumían el $44,44 \%$, luego el $34,72 \%$; y Nunca antes el 30,56\% y posteriormente el $51,39 \%$. 


\section{Gráfico 4.}

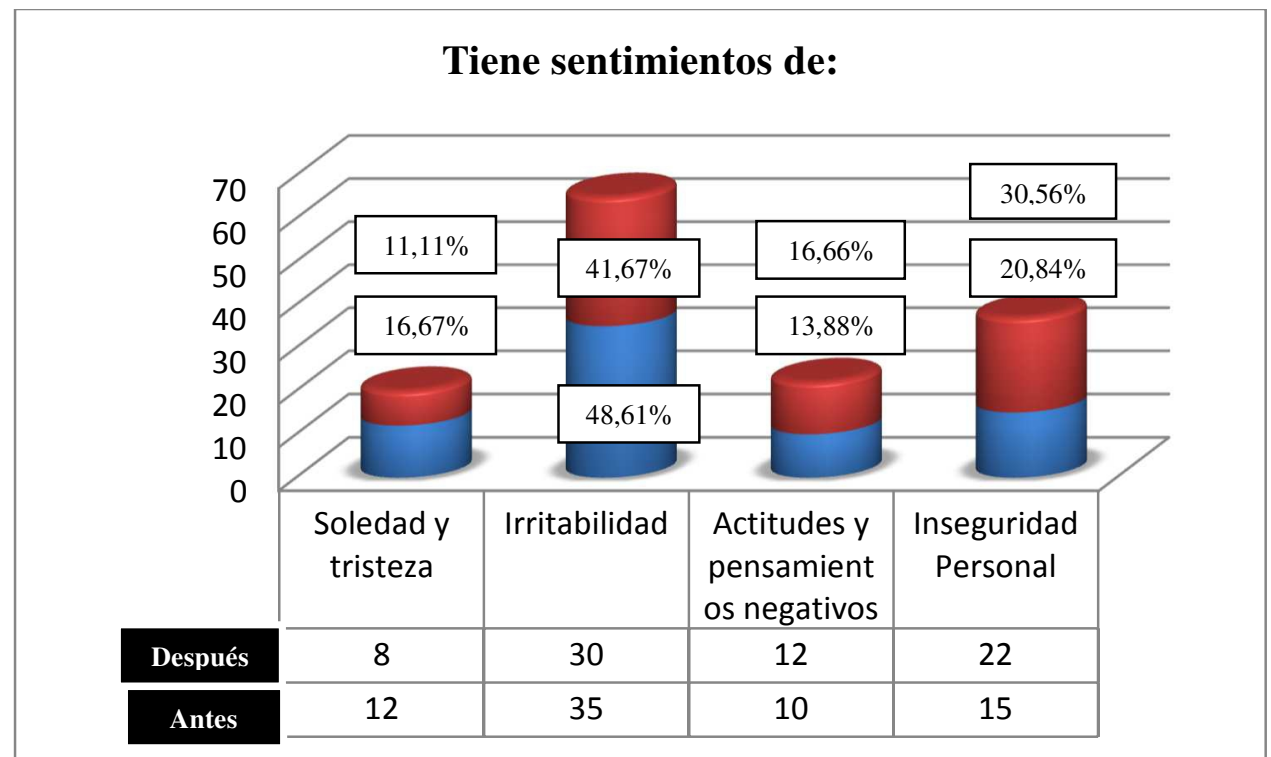

Fuente: Encuesta aplicada a los estudiantes de la Carrera de Cultura Física y Entrenamiento Deportivo de UNACH

Realizado por: equipo de investigación

\section{Análisis}

Referente a los sentimientos de soledad y tristeza, antes el $16,67 \%$ y posterior a la Intervención el 11,11\%; Irritabilidad, antes el 48,61\% y después el 41,67\%; sobre Actitudes y pensamientos negativos, antes el $13,88 \%$ y luego el 16,66\%; y la Inseguridad personal, antes el 20,84\% y posterior el $30,56 \%$. 
Gráfico 5 .

\section{En la jornada académica presenta dolores de:}

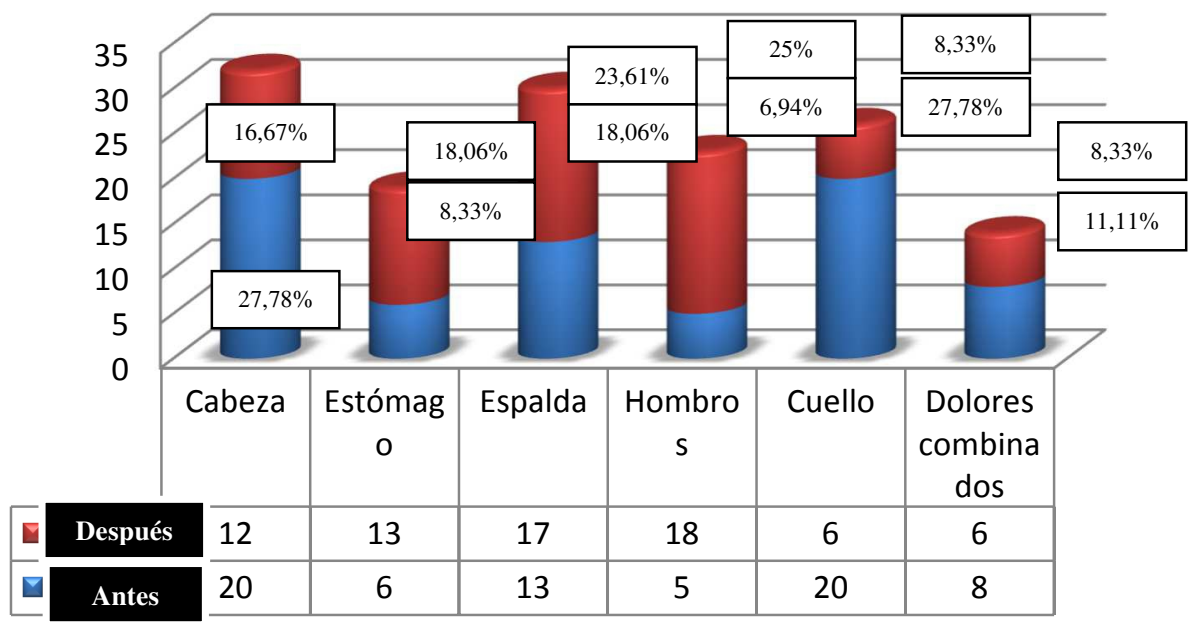

Fuente: Encuesta aplicada a los estudiantes de la Carrera de Cultura Física y Entrenamiento Deportivo de la UNACH

Realizado por: equipo de investigación

\section{Análisis}

En cuanto a los dolores permanentes durante la Jornada Académica, los dolores de cabeza antes de la Intervención Psicopedagógica era de 27,78\%, después el 16,67\%, el dolor de estómago antes el 8,33\%, después el 18,06\%; Dolor de espalda, antes 18,06\%, después el 23,61\%; Dolor de hombros, antes el 6,94\%, después el 25\%; Dolor de cuello, antes el 27,78\% después el 8,33\%; y Dolores combinados antes el 11,11\% y posteriormente el $8,33 \%$. 
Gráfico 6.

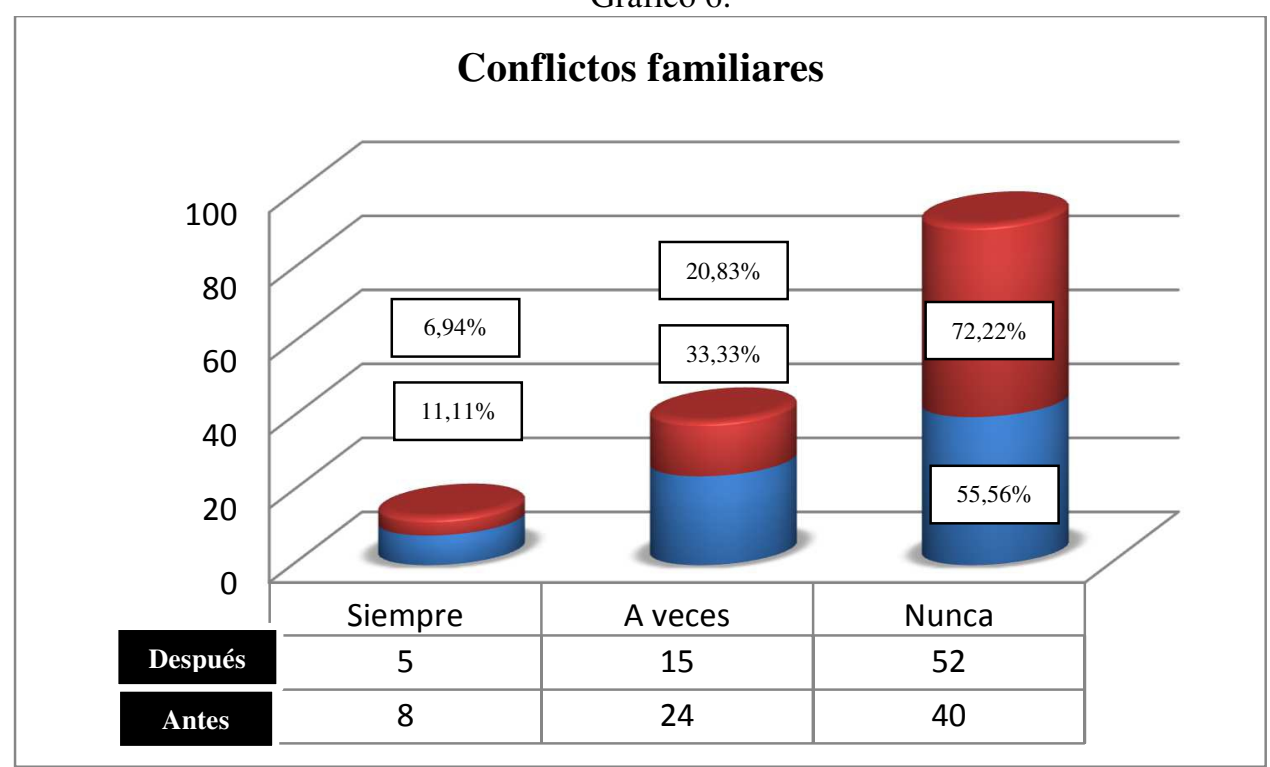

Fuente: Encuesta aplicada a los estudiantes de la Carrera de Cultura Física y Entrenamiento Deportivo de la UNACH

Realizado por: equipo de investigación

\section{Análisis}

En lo que respecta a los conflictos familiares, estudiantes que Siempre lo tenían el 11,11\% y luego de la Intervención Psicopedagógica el $6,94 \%$; A veces, antes el 33,33\%, después el 20,83\%; Nunca antes el $55,56 \%$ y después el $72,22 \%$. 
$\underline{\text { Factores académicos }}$

Gráfico 7.

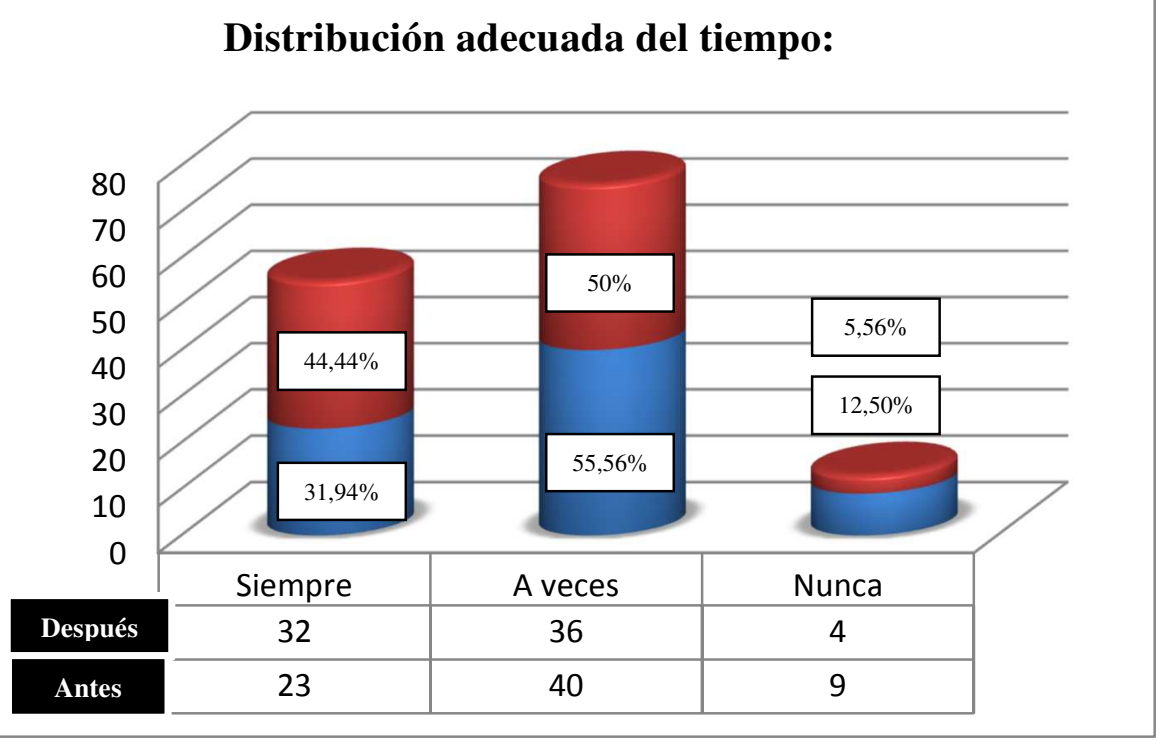

Fuente: Encuesta aplicada a los estudiantes de la Carrera de Cultura Física y Entrenamiento Deportivo de la UNACH

Realizado por: equipo de investigación

\section{Análisis}

En cuanto a la distribución del tiempo referente a sus estudios, Siempre el 31,94\%, luego de los talleres de Intervención el 44,44\%; A veces antes el 55,56\%, después el 50\%; Y Nunca antes el 12,50\% y después el $5,56 \%$. 
Gráfico 8.

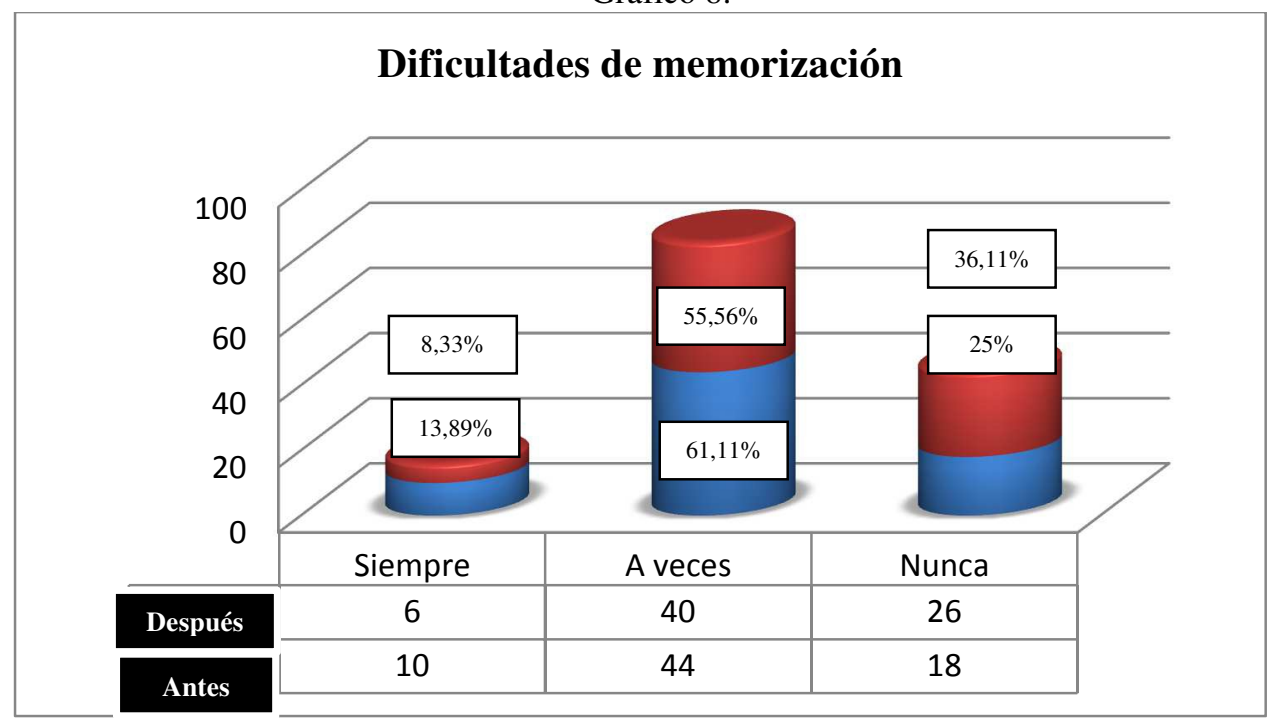

Fuente: Encuesta aplicada a los estudiantes de la Carrera de Cultura Física y Entrenamiento Deportivo de la UNACH

Realizado por: equipo de investigación

\section{Análisis}

Referente a su proceso de memorización, los estudiantes Siempre tenían dificultades el 13,89\%, después de la Intervención el 8,33\%; A veces antes el $61,11 \%$ y después el $55,56 \%$; y Nunca antes el 25\% y después el $36,11 \%$.

Tabla 16. Promedios de los Records Académicos

\begin{tabular}{|c|c|c|}
\hline Rangos & Fa & Fr \\
\hline $10-9.0$ & 6 & $6,19 \%$ \\
\hline $8.99-8$ & 19 & $19,59 \%$ \\
\hline $7.99-7$ & 33 & $34,02 \%$ \\
\hline $6.99-6$ & 38 & $39,17 \%$ \\
\hline $5.99-5$ & 1 & $1,03 \%$ \\
\hline TOTAL & 97 & $100 \%$ \\
\hline
\end{tabular}

Fuente: Secretaría de la Carrera de Cultura Física y Entrenamiento Deportivo de la UNACH Autor: César Mario Rodríguez Sotomayor. 
Gráfico 9.

\section{PROMEDIOS DE RECORDS ACADÉMICOS}

$\square 10-9.0 \quad \square 8.99-8 \quad \square 7.99-7 \quad \square 6.99-6 \quad \square 5.99-5$

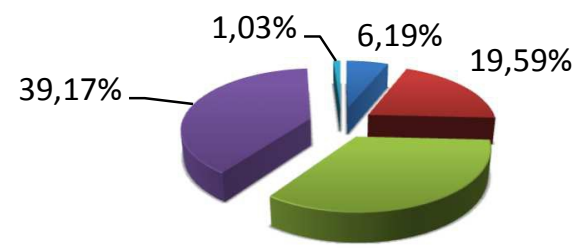

$34,02 \%$

Fuente: Secretaría de la Carrera de Cultura Física y Entrenamiento Deportivo de la Universidad Nacional de Chimborazo.

Realizado por: equipo de investigación

\section{Interpretación}

Es el conjunto de las materias aprobadas, las calificaciones y los niveles que cursa. Toda la información académica importante que se requiere acumular a manera de un historial académico que permita la consolidación de etapas sucesivas, para posteriores trámites administrativos.

Tabla 17. Promedios después del Programa de Intervención

\begin{tabular}{|c|c|c|}
\hline Rangos & Fa & Fr \\
\hline $10-9.0$ & 5 & $6,94 \%$ \\
\hline $8.99-8$ & 8 & $11,11 \%$ \\
\hline $7.99-7$ & 47 & $65,28 \%$ \\
\hline $6.99-6$ & 8 & $11,11 \%$ \\
\hline $5.99-5$ & 4 & $5,56 \%$ \\
\hline TOTAL & 72 & $100,00 \%$ \\
\hline
\end{tabular}

Fuente: Secretaría de la Carrera de Cultura Física y Entrenamiento Deportivo de la UNACH Realizado por: equipo de investigación Gráfico 10.

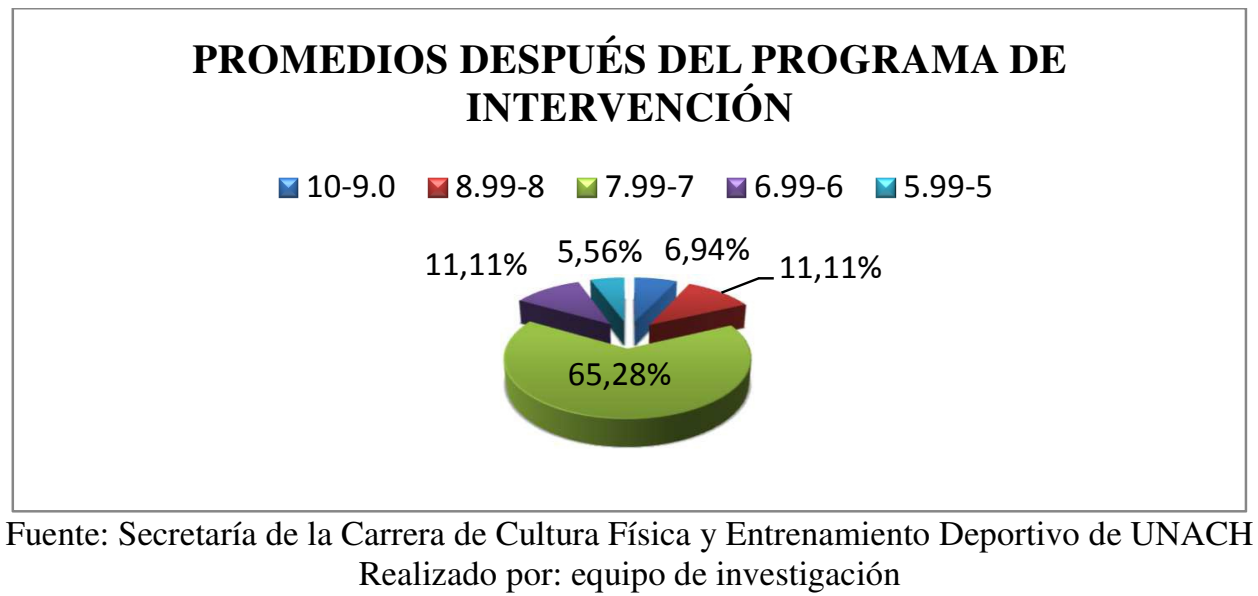




\section{Interpretación}

El promedio es el resultado que se obtiene al dividir la suma de varias cantidades por el número de sumandos.

Gráfico 11.

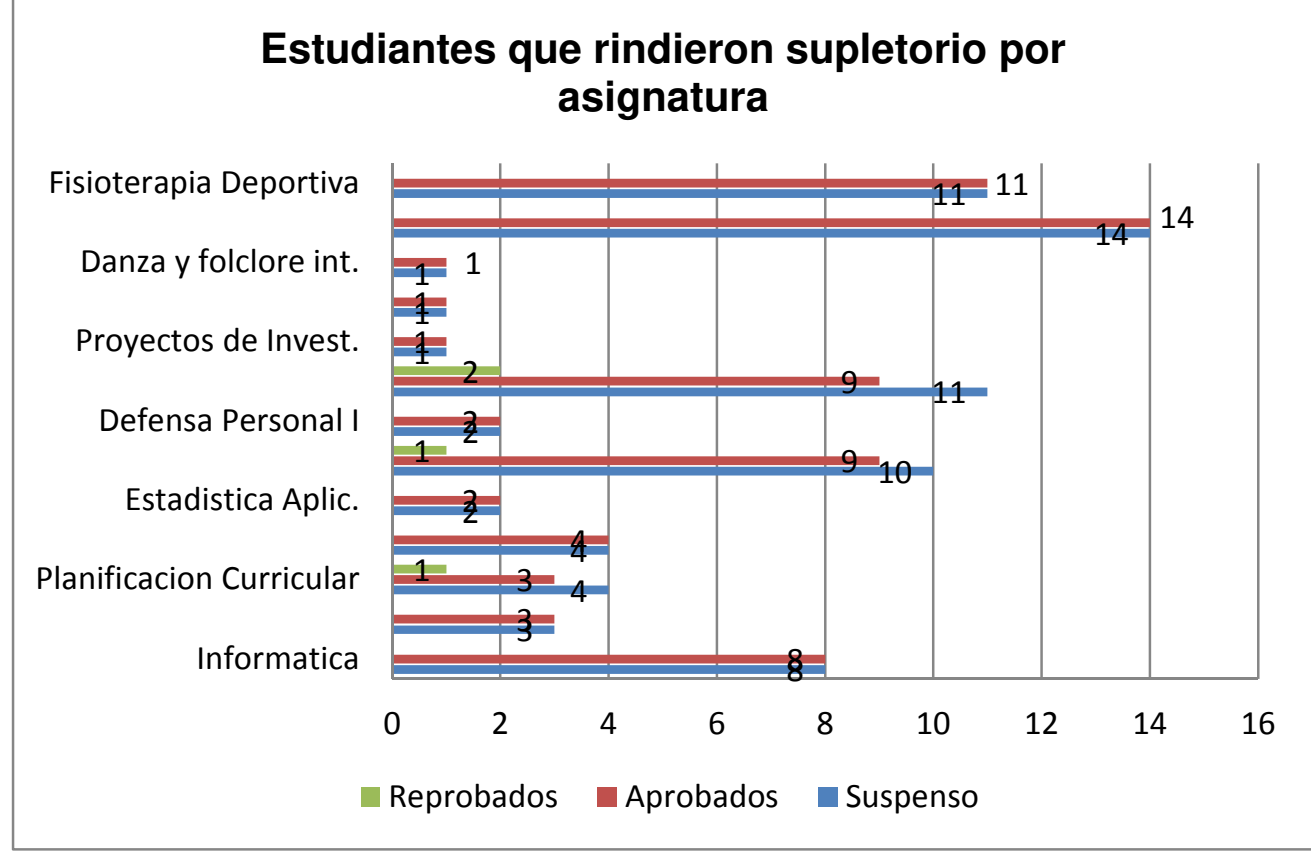

Fuente: Secretaria de Carreras de la Facultad de Ciencias de la Salud de la Universidad Nacional de Chimborazo.

Realizado por: equipo de investigación

\section{Comprobación de Hipótesis}

\section{Hipótesis de Investigación}

Hi: Los factores psicosociales inciden significativamente en el Rendimiento Académico de los estudiantes de la carrera de Cultura Física y Entrenamiento Deportivo de la Universidad Nacional de Chimborazo.

\section{Hipótesis Nula}

Ho: Los factores psicosociales NO inciden en el Rendimiento Académico de los estudiantes de la carrera de Cultura Física y Entrenamiento Deportivo de la Universidad Nacional de Chimborazo.

La hipótesis de investigación será comprobada a través de las hipótesis de trabajo utilizando el estadístico Chi-cuadrado $\left(\chi^{2}\right)$.

\section{Comprobación de la Hipótesis Específica 1}

H1: Los factores psicológicos inciden significativamente en el bajo rendimiento académico de la población estudiantil de la carrera de Cultura Física y Entrenamiento Deportivo. 
Tabla De Frecuencias Observadas

\begin{tabular}{|c|c|c|c|}
\hline \multirow{2}{*}{$\begin{array}{c}\text { FACTORES } \\
\text { PSICOLÓGICOS }\end{array}$} & \multicolumn{2}{|c|}{ RENDIMIENTO ACADÉMICO } & \multirow{2}{*}{ TOTAL } \\
\cline { 2 - 3 } & BAJO & ALTO & \\
\hline SI & 12 & 16 & 28 \\
\hline NO & 40 & 4 & 44 \\
\hline TOTAL & 52 & 20 & 72 \\
\hline
\end{tabular}

TABLA DE FRECUENCIAS ESPERADAS

\begin{tabular}{|c|c|c|c|}
\hline FACTORES PSICOLÓGICOS & \multicolumn{2}{|c|}{ RENDIMIENTO ACADÉMICO } & \multirow{2}{*}{ TOTAL } \\
\cline { 2 - 3 } & BAJO & ALTO & 28 \\
\hline SI & 20,22 & 7,78 & 44 \\
\hline NO & 31,78 & 12,22 & 72 \\
\hline TOTAL & 52 & 20 & \\
\hline
\end{tabular}

\section{CHI-CUADRADO CALCULADO}

\begin{tabular}{|c|c|c|c|c|} 
fo & $\boldsymbol{F e}$ & $\boldsymbol{f o}-\boldsymbol{f e}$ & $\left(f_{o}-f_{\theta}\right)^{2}$ & $\frac{\left(f_{o}-f_{\theta}\right)^{2}}{f_{\theta}}$ \\
\hline 12 & 20,22 & $-8,22$ & 67,5684 & 33,015 \\
\hline 16 & 7,78 & 8,22 & 67,5684 & 8,685 \\
\hline 40 & 31,78 & 8,22 & 67,5684 & 2,126 \\
\hline 4 & 12,22 & $-8,22$ & 67,5684 & 5,529 \\
\hline & & & & 49,355 \\
\hline
\end{tabular}

En consecuencia: Chi-Cuadrado calculado $\left(x_{c}{ }^{2}\right)$

$$
x_{c}{ }^{2}=\sum \frac{(f o-f e)^{2}}{f e}=49,355
$$

- $\quad$ Nivel de significación: $\alpha=0,05$

- $\quad$ Grados de libertad: $(2-1) \cdot(2-1)=1$

El chi-cuadrado teórico con un nivel de significación $\alpha=0,05$ y 1 grado de libertad es $\mathbf{3 , 8 4}$.

Zona de aceptación de 


\section{Decisión}

Como el Chi-chadrado calculado ( $\left.x_{c}{ }^{2}=49,355\right)$, es mayor que el Chi-cuadrado teórico $\left(x_{t}{ }^{2}=3,84\right)$, se rechaza la hipótesis nula y en consecuencia se acepta la hipótesis de investigación, esto es:

Los factores psicológicos inciden significativamente en el bajo rendimiento académico de la población estudiantil de la carrera de Cultura Física y Entrenamiento Deportivo.

\section{Comprobación de la Hipótesis Específica 2}

H2: Los factores sociales inciden significativamente en el bajo rendimiento académico de la población estudiantil de la carrera de Cultura Física y Entrenamiento Deportivo.

Tabla De Frecuencias Observadas

\begin{tabular}{|c|c|c|c|}
\hline \multirow{2}{*}{$\begin{array}{c}\text { FACTORES } \\
\text { SOCIALES }\end{array}$} & \multicolumn{2}{|c|}{ RENDIMIENTO ACADÉMICO } & \multirow{2}{*}{ TOTAL } \\
\cline { 2 - 3 } & BAJO & ALTO & \\
\hline SI & 14 & 15 & 29 \\
\hline NO & 38 & 5 & 43 \\
\hline TOTAL & 52 & 20 & 72 \\
\hline
\end{tabular}

Tabla De Frecuencias Esperadas

\begin{tabular}{|c|c|c|c|}
\hline \multirow{2}{*}{$\begin{array}{c}\text { FACTORES } \\
\text { PSICOLÓGICOS }\end{array}$} & \multicolumn{2}{|c|}{ RENDIMIENTO ACADÉMICO } & \multirow{2}{*}{ TOTAL } \\
\cline { 2 - 3 } & BAJO & ALTO & \\
\hline SI & 20,94 & 8,06 & 27 \\
\hline NO & 31,06 & 11,94 & 45 \\
\hline TOTAL & 52 & 20 & 72 \\
\hline
\end{tabular}

Chi-Cuadrado Calculado

\begin{tabular}{|c|c|c|c|c|}
\hline \multirow{2}{*}{ fo } & $\mathrm{Fe}$ & fo $-\mathrm{fe}\left(f_{o}-f_{e}\right)^{2}$ & $\frac{\left(f_{o}-f_{e}\right)^{2}}{f_{e}}$ \\
\hline 14 & 20,94 & $-6,94$ & 48,1636 & 2,30 \\
\hline 15 & 8,06 & 6,94 & 48,1636 & 5,98 \\
\hline 38 & 31,06 & 6,94 & 48,1636 & 1,55 \\
\hline 5 & 11,94 & $-6,94$ & 48,1636 & 4,03 \\
\hline & & & & 13,86 \\
\hline
\end{tabular}

En consecuencia: Chi-Cuadrado calculado $\left(x_{c}{ }^{2}\right)$

$$
x_{c}{ }^{2}=\sum \frac{(f o-f e)^{2}}{f e}=13,86
$$

- $\quad$ Nivel de significación: $\alpha=0,05$

- $\quad$ Grados de libertad: $(2-1) \cdot(2-1)=1$

El chi-cuadrado teórico con un nivel de significación $\alpha=0,05$ y 1 grado de libertad es $\mathbf{3 , 8 4}$. 


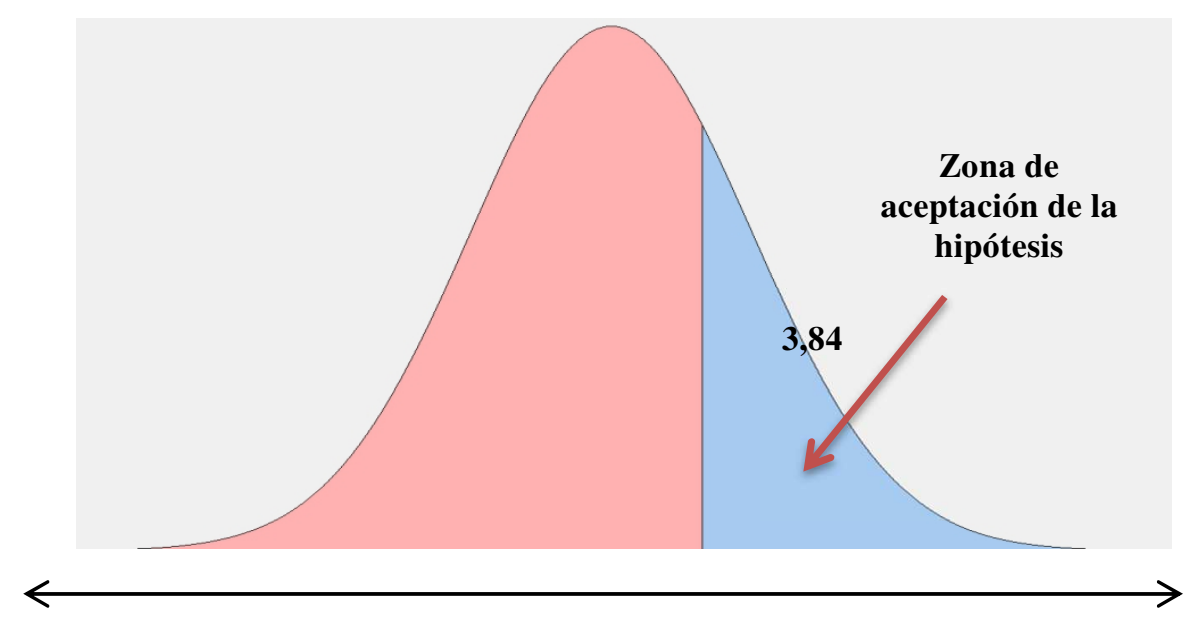

\section{Decisión}

Como el Chi-cuadrado calculado $\left(x_{c}{ }^{2}=13,86\right)$, es mayor que el Chi-cuadrado teórico $\left(x_{t}^{2}=3,84\right)$, se rechaza la hipótesis nula y en consecuencia se acepta la hipótesis de investigación, esto es:

Los factores sociales inciden significativamente en el bajo rendimiento académico de la población estudiantil de la carrera de Cultura Física y Entrenamiento Deportivo.

\section{Comprobación de la Hipótesis Específica 3}

H3: Los Programas de Intervención Psicopedagógica mejoran significativamente el rendimiento académico de la población estudiantil de la carrera de Cultura Física y Entrenamiento Deportivo.

Tabla De Frecuencias Observadas

\begin{tabular}{|c|c|c|c|c|c|}
\hline \multirow{2}{*}{$\begin{array}{c}\text { PROGRAMAS DE } \\
\text { INTERVENCIÓN } \\
\text { PSICOPEDAGÓGICA }\end{array}$} & \multicolumn{4}{|c|}{ MEJORA DEL RENDIMIENTO ACADÉMICO } & \multirow[b]{2}{*}{ TOTAL } \\
\hline & SIEMPRE & $\begin{array}{c}\text { CASI } \\
\text { SIEMPRE }\end{array}$ & $\begin{array}{c}\text { A } \\
\text { VECES }\end{array}$ & NUNCA & \\
\hline SI & 12 & 8 & 6 & 2 & 28 \\
\hline NO & 5 & 7 & 17 & 15 & 44 \\
\hline TOTAL & 17 & 15 & 23 & 17 & 72 \\
\hline
\end{tabular}

Tabla De Frecuencias Esperadas

\begin{tabular}{|c|c|c|c|c|c|}
\hline \multirow{2}{*}{$\begin{array}{c}\text { PROGRAMAS DE } \\
\text { INTERVENCIÓN } \\
\text { PSICOPEDAGÓGICA }\end{array}$} & \multicolumn{4}{|c|}{ MEJORA DEL RENDIMIENTO ACADÉMICO } & \multirow[b]{2}{*}{ TOTAL } \\
\hline & SIEMPRE & $\begin{array}{c}\text { CASI } \\
\text { SIEMPRE }\end{array}$ & $\begin{array}{c}\text { A } \\
\text { VECES }\end{array}$ & NUNCA & \\
\hline SI & 6,61 & 5,83 & 8,94 & 6,61 & 28 \\
\hline $\mathrm{NO}$ & 10,39 & 9,17 & 14,06 & 10,39 & 44 \\
\hline TOTAL & 17 & 15 & 23 & 17 & 72 \\
\hline
\end{tabular}


Chi-Cuadrado Calculado

\begin{tabular}{|c|c|c|c|c|}
\hline fo & fe & fo $-\mathrm{fe}\left(f_{o}-f_{e}\right)^{2}$ & $\frac{\left(f_{o}-f_{e}\right)^{2}}{f_{e}}$ \\
\hline 12 & 6,61 & 5,39 & 29,0521 & 4,3952 \\
\hline 8 & 5,83 & 2,17 & 4,7089 & 0,8077 \\
\hline 6 & 8,94 & $-2,94$ & 8,6436 & 0,9668 \\
\hline 2 & 6,61 & $-4,61$ & 21,2521 & 3,2551 \\
\hline 5 & 10,39 & $-5,39$ & 29,0521 & 2,7962 \\
\hline 7 & 9,17 & $-2,17$ & 4,7089 & 0,5135 \\
\hline 17 & 14,06 & 2,94 & 8,6436 & 0,6148 \\
\hline 15 & 10,39 & 4,61 & 21,2521 & 2,0454 \\
\hline & & & & 15,3947 \\
\hline
\end{tabular}

En consecuencia: Chi-Cuadrado calculado $\left(x_{c}{ }^{2}\right)$

$$
x_{c}^{2}=\sum \frac{(f o-f e)^{2}}{f e}=15,3947
$$

- $\quad$ Nivel de significación: $\alpha=0,05$

- $\quad$ Grados de libertad: $(2-1) .(4-1)=3$

El chi-cuadrado teórico con un nivel de significación $\alpha=0,05$ y 3 grados de libertad es $\mathbf{7 , 8 1}$.

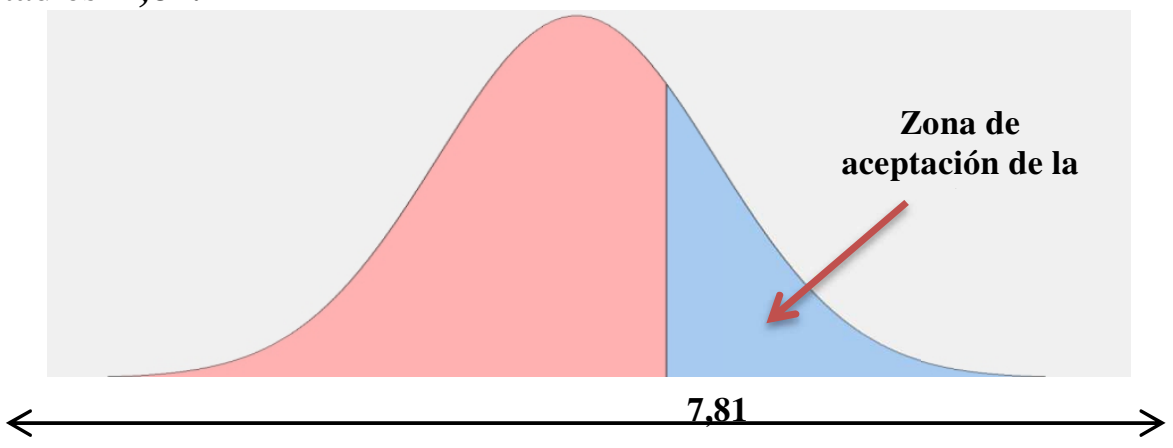

\section{Decisión}

Como el Chi-cuadrado calculado ( $\left.x_{c}{ }^{2}=15,3947\right)$, es mayor que el Chi-cuadrado teórico $\left(x_{t}^{2}=7,81\right)$, se rechaza la hipótesis nula y en consecuencia se acepta la hipótesis de investigación esto es:

Los Programas de Intervención Psicopedagógica mejoran significativamente el rendimiento académico de la población estudiantil de la carrera de Cultura Física y Entrenamiento Deportivo.

\section{Conclusion}

Entre los factores que se evidenciaron en la investigación, se determinó que los factores psicológicos prevalentes que se hallan influyendo 
en el rendimiento académico de los estudiantes, fueron: el insomnio con un 23,61\%, el consumo de alcohol con un 20,83\%, la tristeza, la autoestima, síntomas de depresión, ansiedad, insomnio y las adicciones preferentemente respecto a alcoholismo y tabaquismo.

Se determinó que los principales factores sociales que repercuten en el rendimiento académico de los estudiantes, son la familia, los conflictos familiares, la migración, añadido a esto factores de tipo económico.

Se concluyó que según sus récords académicos, la media es de $7,05 / 10$, lo cual nos permitió establecer de que su rendimiento se halla al límite, razón por la cual durante el período académico de Septiembre 2014 Febrero 2015 de toda la carrera, es decir de los siete semestres con que cuenta, 72 estudiantes se quedaron a rendir una prueba supletoria, siendo más prevalentes en asignaturas como Gerencia Deportiva I (14), Fisioterapia Deportiva (11), Danza y Folclore Nacional (11), Medicina Deportiva (10) y otras asignaturas en menor número.

Se elaboró un Programa de Intervención Psicopedagógica con el fin de proporcionarles la ayuda pertinente dentro del ámbito psicológico y pedagógico, aspectos que les permitió rendir de forma satisfactoria sus evaluaciones supletorias.

Se evaluó el Programa de Intervención Psicopedagógica con entera satisfacción, puesto que de los 72 estudiantes que rindieron la prueba supletoria, apenas 4 estudiantes no lograron superarla, 2 estudiantes en la asignatura de Danza y Folclore, 1 estudiante en Medicina Deportiva y 1 estudiante en Planificación Curricular.

\section{References:}

1. Abarca, A., \& Sánchez, M. (2005). Deserción Estudiantil en la Educación Superior: El caso de la Universidad de Costa Rica. Revista Electrónica Actualidades Investigativas en Educación, 5, 1-22.

2. AGO.USB. 2006. Aspecto trascendental que plantea la Psicopedagogía: el fortalecimiento de las capacidades. ISSN: 16578031. Vol.6 N.2. pág. 147-315. Medellín-Colombia.

3. Armenta, N., Pacheco, C., \& Pineda, E. (2008). Factores Socioeconómicos que intervienen en el Desempeño Académico de los Estudiantes Universitarios de la Facultad de Ciencias Humanas de la Universidad Autónoma de Baja California. Revista IIPSI, 11(1), 153-165.

4. Ausubel P. et al. (1991), Psicología educativa. Un punto de vista cognoscitivo, México, Trillas.

5. Cascón, I. 2000. Predictores del rendimiento académico en alumnos de primero y segundo de BUP. In: Disponible en URL: 
http://www3.usal.es/ inico/investigacion/jornadas/ jornada2/común/c19.html].

6. CONESUP (2015). Consejo Nacional de Educación Superior. Disponible en URL: http://www.ces.gob.ec/index.php?option=com_sobipro\&sid=68:Instit uciones-de-Educacion-Superior\&Itemid $=0$

7. Coon, D., \& Mitterer, J. (2010). Introducción a la Psicología. El acceso a la mente y la conducta. México. CENGAGE Learning.

8. Fernández, O., Martínez, M., \& Melipillán, R. (2009). Estrategias de aprendizaje y autoestima. Su relación con la permanencia y deserción universitaria. Estudios Pedagógicos, XXXV (1), 27-45

9. Sauceda, J. \& Maldonado, J. (2003). Organización Panamericana de Salud: La Familia su Dinámica y Tratamiento. Editorial. IMSS. México.

10. Sierra, R. (1994). Técnicas de investigación Social. 9a. Edición. Madrid. Editorial Paraninfo S. A. 705p. ISBN 84-283-1548-5

11. Vygotsky, L. (1991). Problemas teóricos y metodológicos de la psicología. Madrid: Visor. 